\title{
Large Deformation Behavior of High Strength Steel Under Extreme Loading Conditions: High Temperature and High Strain Rate Experiments and Modeling
}

\author{
Xueyang Li ${ }^{1, *}$, Christian C. Roth ${ }^{1,2}$, and Dirk Mohr ${ }^{1,2}$ \\ ${ }^{1}$ Department of Mechanical and Process Engineering, ETH Zurich, Switzerland \\ ${ }^{2}$ Impact and Crashworthiness Lab, Department of Mechanical Engineering, Massachusetts Institute of Technology, USA
}

\begin{abstract}
Plasticity and fracture experiments are carried out on flat smooth and notched tensile specimens extracted from DP800 steel sheets. A split Hopkinson pressure bar testing system equipped with a load inversion device is utilized to reach high strain rates. Temperature dependent experiments ranging from $20^{\circ} \mathrm{C}$ to $300^{\circ} \mathrm{C}$ are performed at quasi-static strain rates. The material exposes a monotonic strain hardening behaviour with a non-monotonic temperature dependency. The rate-independent material behaviour at room-temperature is described with a non-associated Hill'48 plasticity model and an Swift-Voce strain hardening. A machine learning based model is used multiplicatively to capture the rate and temperature responses. A good agreement between measured and simulated force-displacement curves as well as local surface is obtained. The loading paths to fracture are then extracted to facilitate further development of a temperature dependent fracture initiation model.
\end{abstract}

\section{Introduction}

The use of advanced high strength steels (AHSS) is essential in automotive engineering. This choice is driven by the quest for weight reduction to improve vehicle dynamics, fuel efficiency and cost efficiency [1]. During stamping process and accidental crash loading, strain rate as high as 1000/s can be reached. Subsequent fracture initiation preceded by localized necking is witnessed [2]. It is a common assumption that fracture initiates at a point where weighted measure of accumulated plastic strain reaches a critical value. As direct measurement of strain inside a localized neck prove to be experimentally unfeasible, a plasticity model that is accurate at large strains and captures rate-and temperature effects is demanded.

The wide variety of existing visco-plasticity models can be classified into physics-based models [3] and phenomenological models. Phenomenological constitutive functions are widely implemented in numerical models, especially in finite element simulations used for metal forming [4]. Many established phenomenological models for dynamic loading conditions are based upon the Johnson-Cook model [5], which multiplicatively decomposes the flow stress into three terms that depend on the strain, strain rate and temperature, respectively. The simple J-C plasticity model has been reported to offer reasonable predictions of temperature dependent visco-plasticity response of many metals up to large strains ([5] [6] [7]). These constitutive equations describe explicitly the relations between stress, strain, strain rate and temperature, but are usually limited to their specific deformation mechanism as well as mathematical formulations. For example, the $\mathrm{J}-\mathrm{C}$ temperatures and strain rate formulation break down in case of negative strain rate sensitivity and non-monotonic temperature dependency. Additional model parameters and modified formulation is often required to overcome these limitations. Some researchers have also introduced neural network as a universal function approximator in their constitutive models to describe the rheological behaviour [8] and flow behaviour [4] of metals.

In the present paper, low, intermediate and high strain rate experiments have been performed on flat specimens with notches. We have also performed quasistatic experiments with notched specimen at elevated temperatures. It has been witnessed in our experiments that many dual phase steels exhibit non-monotonic temperature dependency. To accurately determine the strain to fracture inside the necking region, a neural network based term is combined to the mixed SwiftVoce hardening function and a non-associated anisotropic flow rule. The model parameters are identified through an inverse training scheme based on back-propagation [9]. The loading path to fracture are then reported for all experiments, including the stress triaxiality, Lode angle parameter, strain rate, temperature and equivalent plastic strain.

\section{Experiments}

Corresponding author: lixue@ethz.ch 


\subsection{Material, specimens and experimental procedures}

The tested material is a $1.6 \mathrm{~mm}$ thick annealed DP800 sheet. Uniaxial tension (UT) and notched tension (NT) specimens (Fig. 1) are extracted from these sheets. A random speckle pattern is spray painted onto the specimens to facilitate digital image correlation (VIC2D, Correlated Solutions). The logarithmic axial surface strain is measured from relative vertical displacement of two $1 \mathrm{~mm}$ separated points located at the centre of gauge section (red dots in Fig. 1). Experiments at low and intermediate strain rates are conducted on a hydraulic universal testing machine (Instron Model 8801). All experiments at high strain rates are conducted on a split Hopkinson pressure bar (SHPB) system with a specially designed load inversion device for tensile loading [10].

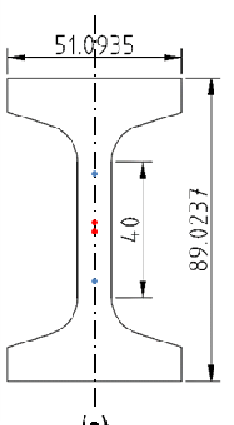

(a)

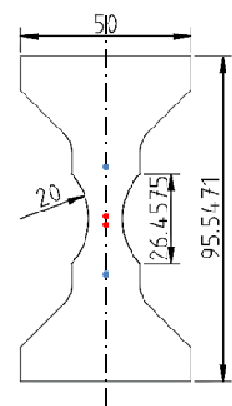

(b)

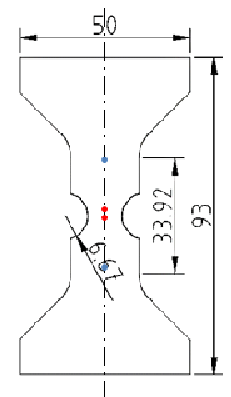

(c)
Fig. 1. Specimen geometries: (a) uniaxial tension specimen (b) NT20 specimen and (c) NT6 specimen.

\subsection{Experimental results}

The yield stress $\sigma_{Y S}$, the ultimate tensile strength $\sigma_{U T S}$, the engineering strains at ultimate tensile strength $\varepsilon_{U}$, the engineering strain to fracture $\varepsilon_{T}$ and Lankford ratios $\gamma_{a}$ are measured from uniaxial tensile tests. The corresponding Lankford ratios suggest direction dependency in both materials.

Figure 2 shows representative force-displacement curves for DP800 steel with NT20 specimens at three different loading speeds. All experiments feature a force maximum before fracture occurs, which increases as a function of the loading speed. After the force maximum, the decrease in axial force per applied displacement increment is higher for faster loading speeds. In addition, a slight increase in displacement to fracture is observed in experiments at high loading speed.

Figure 3 shows representative force-displacement curves with NT20 experiments at 6 different temperatures, together with corresponding maximum force and force at onset of fracture (Fig. 4). Counter intuitively neither force maximum nor failure force show monotonic temperature dependency. The shapes of the force-displacement curves change significantly for high temperature experiments, which indicates a change in hardening behaviour.

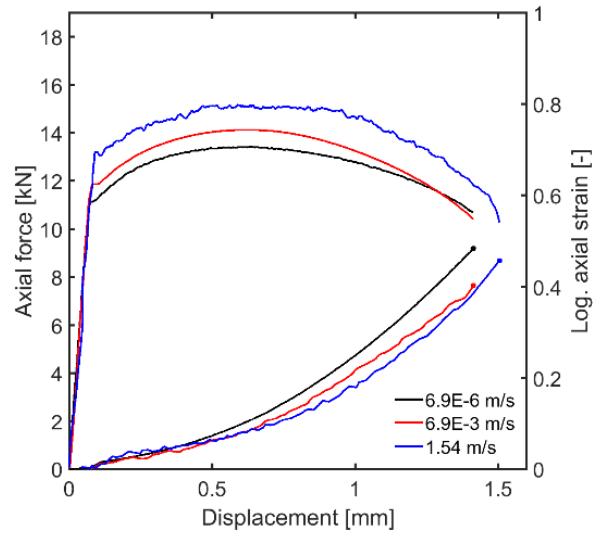

Fig. 2. Force-displacement curve for NT20 specimen at different loading speeds (DP800).

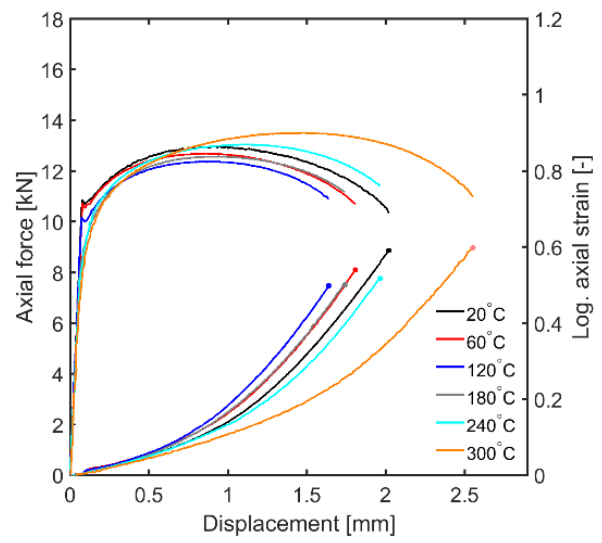

Fig. 3. Force-displacement curve with NT20 specimen for DP800 at different temperatures.

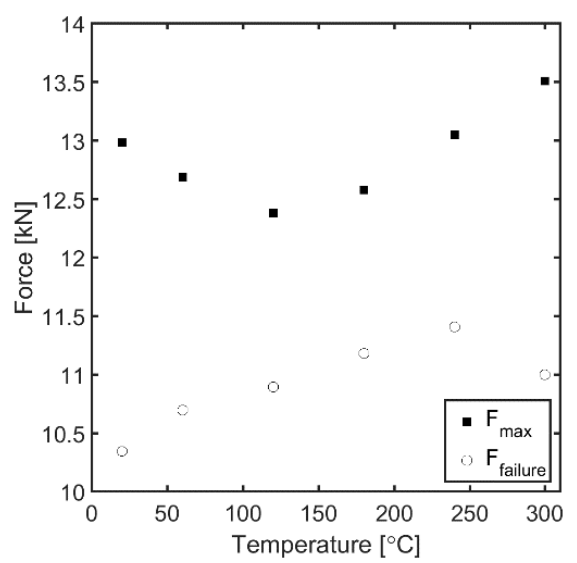

Fig. 4. The corresponding maximum force and force at failure with NT20 tests at elevated temperatures.

\section{Constitutive model}

\subsection{Yield function and flow rule}

The yield function is formatted as:

$$
f[\sigma, k]=\bar{\sigma}-k=0
$$


$\bar{\sigma}$ the von Mises equivalent stress can be expressed as a linear transformation of Cauchy stress vector where $\boldsymbol{P}$ is the positive definite fourth-order symmetric weighting tensor containing Hill constants:

$$
\begin{gathered}
\bar{\sigma}[\boldsymbol{\sigma}]=\sqrt{(\boldsymbol{P} \boldsymbol{\sigma}) \cdot \boldsymbol{\sigma}} \\
\boldsymbol{\sigma}=\left[\begin{array}{llllll}
\sigma_{11} & \sigma_{22} & \sigma_{33} & \sigma_{12} & \sigma_{23} & \sigma_{13}
\end{array}\right]^{T}
\end{gathered}
$$

A non-associated flow potential is introduced utilizing Hill'48

$$
g[\sigma]=\sqrt{(\boldsymbol{G} \sigma) \cdot \boldsymbol{\sigma}}
$$

with

$$
[\boldsymbol{G}]=\left[\begin{array}{cccccc}
1 & G_{12} & -\left(1+G_{12}\right) & 0 & 0 & 0 \\
G_{12} & G_{22} & -\left(G_{12}+G_{22}\right) & 0 & 0 & 0 \\
-\left(1+G_{12}\right) & -\left(G_{12}+G_{22}\right) & 1+2 G_{12}+G_{22} & 0 & 0 & 0 \\
0 & 0 & 0 & G_{33} & 0 & 0 \\
0 & 0 & 0 & 0 & 3 & 0 \\
0 & 0 & 0 & 0 & 0 & 3
\end{array}\right]
$$

The flow potential reduces to isotropic von Mises equivalent stress when $G_{12}=-0.5, G_{22}=1$ and $G_{33}=3$. The increment in the plastic strain vector $\varepsilon_{p}$ is denoted as:

$$
d \varepsilon_{p}=d \lambda \frac{\partial g[\sigma]}{\partial \sigma}
$$

with the plastic strain vector

$$
\boldsymbol{\varepsilon}_{\boldsymbol{p}}=\left[\begin{array}{llllll}
\varepsilon_{11}^{p} & \varepsilon_{22}^{p} & \varepsilon_{33}^{p} & 2 \varepsilon_{12}^{p} & 2 \varepsilon_{23}^{p} & 2 \varepsilon_{13}^{p}
\end{array}\right]^{T}
$$

\subsection{Deformation resistance}

The deformation resistance is multiplicatively decomposed into a reference mixed Swift-Voce strain hardening function [2] and a neural network that describes strain rate and temperature dependent behaviour.

$$
k\left[\bar{\varepsilon}_{p}, \dot{\bar{\varepsilon}}_{p}, T\right]=k_{S V}\left[\bar{\varepsilon}_{p}\right] k_{N N}\left[\bar{\varepsilon}_{p}, \dot{\bar{\varepsilon}}_{p}, T\right]
$$

A neural network model is introduced to describe the effect of the strain and temperature, i.e. the function $k_{N N}\left[\bar{\varepsilon}_{p}, \dot{\bar{\varepsilon}}_{p}, T\right]$ is approximated through a feed-forward neural network. The analytical formulation for activating the $m^{\text {th }}$ layer of feedforward net with hyperbolic tangent transfer function is:

$$
H_{k}^{(m)}=\tanh \left[\sum_{l=1}^{P} W_{k l}^{(m-1)} H_{l}^{(m-1)}+b_{k}^{(m-1)}\right]
$$

where $\mathbf{W}$ and $\mathbf{b}$ represent the weight matrix and bias vector, respectively.

The network structure and normalization of inputs and output variables are summarized Table 1. A network with 3 hidden layers each containing 10 neurons was selected for this study. Adding input layer, output layer and constant bias nodes, the number of neurons for each layer becomes [4,11,11,11,1], which corresponds to 297 independent weight parameters in total.

For numerical analysis in finite element simulation, temperature is treated as internal state variable. The analysis is switched from isothermal to adiabatic condition by a sigmoid shaped strain rate dependent factor (for details, see [2]).

Table 1. Normalization parameters.

\begin{tabular}{|c|c|}
\hline Inputs & {$\left[\log \left(\bar{\varepsilon}_{p}\right), \log \left(\dot{\bar{\varepsilon}}_{p}\right), T\right]^{T}$} \\
\hline Input range & $\left\{\begin{array}{cc}\left(\begin{array}{ll}-7 & 1\end{array}\right) & N / A \\
\left(\begin{array}{cc}-9 & 10\end{array}\right) & s^{-1} \\
\left(\begin{array}{ll}293 & 593\end{array}\right) & K\end{array}\right\}$ \\
\hline Output & $k_{N N}$ \\
\hline Output range & $(0.5-1.5)$ \\
\hline
\end{tabular}

\section{Model parameter identification}

\subsection{Yield function and strain hardening}

The plasticity model parameters are identified as follows:

- The parameters for anisotropic flow potential are derived from Lankford ratios measured from uniaxial tensile experiments.

- The Swift and Voce strain hardening parameters are identified with least-square fit of the true stress-plastic strain curves from quasi-static uniaxial tensile tests up to the onset of necking.

- The parameter $\alpha$ in mixed Swift-Voce hardening law is identified through inverse analysis of room temperature quasi-static NT20 experiments.

- The parameters $\left\{E, v, \rho, \eta_{k}, \dot{\varepsilon}_{a}\right\}$ are taken from literature.

- The specific heat $C_{p}$ is experimentally determined from differential scanning calorimetry (Mettler Toledo DSC 1).

\subsection{Machine-learning approach to identify rate and temperature effects}

Backpropagation algorithm is used to train the selected multi-layer feed forward neural network. A square error function that evaluates difference between experimental and computed force-displacement curve is chosen:

$$
l_{N j}^{n_{E X P}}=\frac{1}{2}\left(F_{N j}^{N N}-F_{N j}^{E X P}\right)^{2}
$$

Here the error is a function of net tensile force, which can be approximated by the sum of reaction force over all elements on mid-plane:

$$
F_{N j}^{N N} \approx 4 \sum_{n_{E L}} A_{N j}^{n_{E L}} \bar{\sigma}_{N j}^{n_{E L}}
$$

Thus for each element, the partial derivative of error function against network output is: 


$$
\frac{\partial l_{N j}^{n_{E X P}}}{\partial k_{N N}}=4\left(F_{N j}^{N N}-F_{N j}^{E X P}\right) A_{N j}^{n_{E L}}\left(k_{S V}\right)_{N j}^{n_{E L}}
$$

with this partial derivation, we invoke the classic learning rule:

$$
\Delta W_{i j}^{n}=-\eta_{L} \frac{\partial l_{N j}^{n_{E X P}}}{\partial W_{i j}^{n}}
$$

where $\eta_{L}$ is the learning rate. The actual weight update is conducted using resilient back propagation algorithm (RPROP) [9]. Upon completion of weight update, a new Abaqus user subroutine is generated using newly updated network parameters. We then proceed to re-run simulations on all NT20 experiments at various strain rates and elevated temperatures and evaluate new weight update. The inverse training is complete when total error is lower than a set threshold or maximum number of iteration is reached.

\subsection{Comparison of the experimental and simulation results on training dataset}

Figure 5 shows the outcome of plasticity model after this training stage. Recall that these NT20 experiments have been used as training dataset. All computed forcedisplacement curves (solid lines) show good agreement with those measured experimentally (dotted lines). However, a consistent overestimation on the computed local axial strain compared with experimental results can be witnessed.

Figure 6 shows the simulated temperature-strain rate history extracted from sample mid-plane elements of NT20 specimen. Note that the upper right region (high speed test starting at elevated temperatures) of is not fully covered. The loading paths to fracture in terms of the equivalent plastic strain against stress triaxiality and Lode angle parameters are shown in Figure 7.

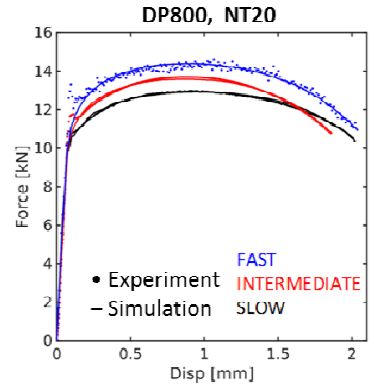

(a)

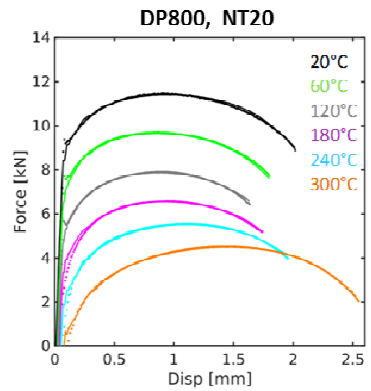

(c)

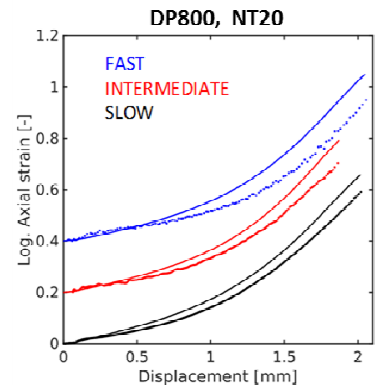

(b)

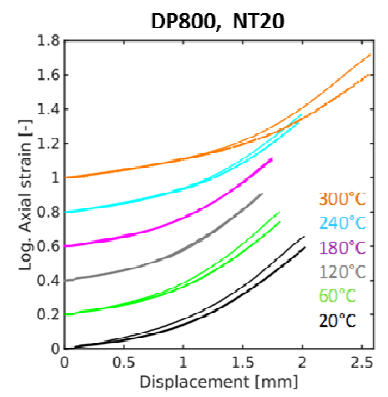

(d)
Fig. 5. Result of final training: experimental and simulated curves with NT20 specimen. Experiments at different strain rates are simulated to generate (a) force-displacement curves (b) local strain histories. Tests at elevated temperatures are shown in (c) force-displacement curves and (d) local strain histories. The temperature force-displacement curves are shifted down by $1.5 \mathrm{kN}$ respectively for better visibility. The axial strain histories are also shifted consecutively upward by 0.2 .

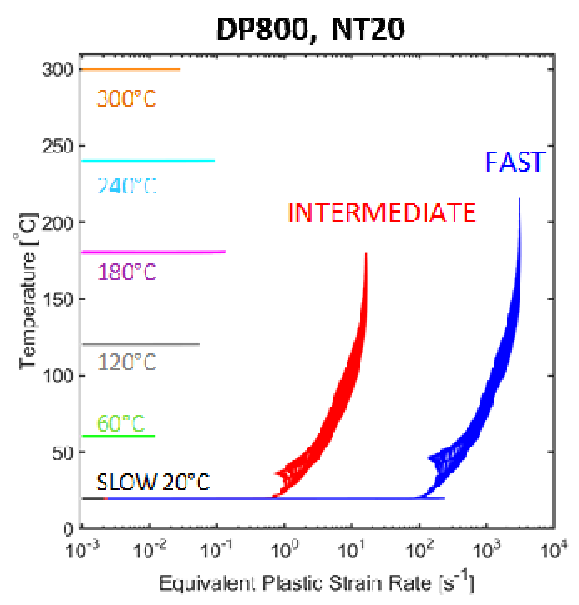

Fig. 6. Loading paths in temperature-strain rate plane for training dataset. 


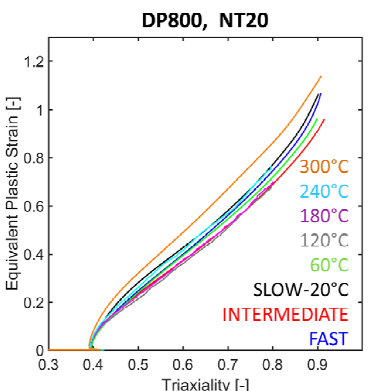

(a)

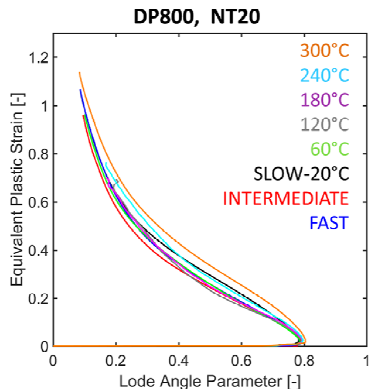

(b)
Fig. 7. Loading path to fracture extracted from NT20 simulation: The evolution of equivalent plastic strain as a function of (a) stress triaxiality, (b) Lode angle parameter.

\section{Model validation}

Experiments with NT6 specimen at different loading speeds serve as validation for the proposed plasticity model. Figure 8 compares the simulated and measured force-displacement curves and evolution of local axial strain for NT6 experiments. The force levels of the simulation and experiment agree well. For intermediate and high strain rate cases, a slight over-estimation on the simulated local surface strain is observed towards the onset of fracture.

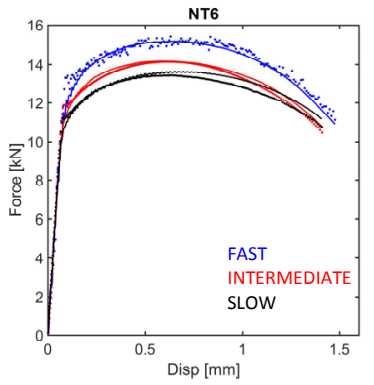

(a)

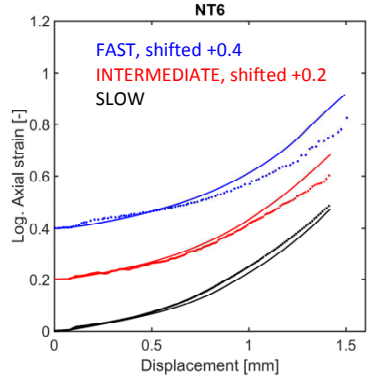

(b)
Fig. 8. Validation dataset: (a) force-displacement and (b) surface strain histories for NT6 specimen at different loading speed. The local axial strain histories are shifted upward for 0.2 consecutively for better visibility.

\section{Conclusions}

An experimental and numerical investigation on the effect of loading speed and temperatures on annealed DP800 is carried out. Experiments on specimen extracted from DP800 steel sheets are conducted to obtain different stress states, strain rates and temperatures. A machine learning based plasticity model is proposed which couples a combined Swift-Voce strain hardening law with a neural network based rate- and temperature dependency. Temperature is treated as internal variable by the model. The chosen plasticity model is identified using a back propagation based RPROP algorithm and showcases fast convergence during calibration (mean error $<0.01 \mathrm{kN}$ in 59 epochs). Reasonable agreement of calculated and measured results is observed for NT20 and NT6 specimen geometries at different temperatures and loading speeds.
The histories of stress triaxiality, Lode angle, equivalent plastic strain, strain rate and temperatures at the location of fracture initiation are then extracted from numerical simulations for further development of a temperature dependent fracture initiation model.

\section{References}

1. M. Dunand and D. Mohr, International Jounral of Solids and Structures, vol. 47, pp. 1130-1143 (2009)

2. C. Roth and D. Mohr, International Journal of Plasticity, vol. 56, pp. 19-44 (2014)

3. A. Khan, M. Baig, S. Choi, H. Yang and X. Sun, International Journal of Plasticity, Vols. 30-31, pp. 1-17 (2012)

4. A. Jenab, S. I. Sari, D. Green, T. Rahmaan and M. Worswick, Materials and Design, vol. 94, pp. 262-273 (2016)

5. G. Johnson and W. Cook, Engineering Fracture Mechanics, vol. 21, no. 1, pp. 31-48 (1985)

6. T. Fras, C. Roth and D. Mohr, International Journal of Impact Engineering, vol. 111, pp. 147-164 (2018)

7. K. Pack and C. Roth, International Journal of Fracture, vol. 198, pp. 197-220 (2016)

8. Y. Lin and X. Chen, Materials and Design, vol. 32, pp. 1733-1759 (2010)

9. M. Riedmiller and H. Braun, IEEE International Conference on Neural Networks, vol. 1, pp. 586--591 (1993)

10. C. Roth, G. Gary and D. Mohr, Experimental Mechanics, vol. 55, pp. 1803-11 (2015) 
